\title{
VIRTUALLY FREE PRO-P PRODUCTS
}

\author{
TH. WEIGEL AND P. A. ZALESSKII
}

\begin{abstract}
It is shown that a finitely generated pro- $p$ group $G$ which is a virtually free pro- $p$ product splits either as a free pro- $p$ product with amalgamation or as a pro- $p$ HNN-extension over a finite $p$-group. More precisely, $G$ is the pro- $p$ fundamental group of a finite graph of finitely generated pro- $p$ groups with finite edge groups. This generalizes previous results of W. Herfort and the second author (cf. [2]).
\end{abstract}

\section{INTRODUCTION}

In 1965, J-P. Serre showed that a torsion free virtually free pro- $p$ group must be free (cf. 7]). This motivated him to ask the question whether the same statement holds also in the discrete context. His question was answered positively some years later. In several papers (cf. [10, 11, [13]), J.R. Stallings and R.G. Swan showed that free groups are precisely the groups of cohomological dimension 1 , and at the same time J-P. Serre himself showed that in a torsion free group $G$ the cohomological dimension of a subgroup of finite index coincides with the cohomological dimension of $G$ (cf. [8]).

One of the major tools for obtaining this type of result - the theory of ends provided deep results also in the presence of torsion. The first result to be mentioned is 'Stallings' decomposition theorem' (cf. [12]). It generalizes the previously mentioned result to virtual free products.

Theorem 1.1 (J.R. Stallings). Let $G$ be a finitely generated group containing a subgroup of finite index which is a non-trivial free product. Then $G$ splits either as a free product with amalgamation or as an $H N N$-extension over a finite group.

The purpose of this paper is to prove a pro- $p$ analogue of Theorem 1.1 .

Theorem A. Let $G$ be a finitely generated pro-p group containing an open subgroup which is a non-trivial free pro-p product. Then $G$ splits either as a free pro-p product with amalgamation or as a pro-p HNN-extension over a finite $p$ group.

In the torsion free case Theorem A yields a splitting of $G$ into a non-trivial free pro- $p$ product.

Corollary B. Let $G$ be a finitely generated torsion free pro-p group which is a virtual free pro-p product. Then $G$ is a non-trivial free pro-p product.

In contrast to the proof of Theorem 1.1 which uses the theory of ends, the proof of Theorem $\mathrm{A}$ is accomplished by using purely combinatorial methods in pro- $p$ group theory, and the description of finitely generated virtually free pro- $p$ groups

Date: September 28, 2018

2010 Mathematics Subject Classification. Primary 20E18, secondary 20E05, 20E06, 20J06. 
obtained by W. Herfort and the second author (cf. [2]). In fact, the techniques of pro- $p$ groups acting on pro- $p$ trees are used in order to obtain the following more conceptual version of Theorem A (cf. Thm. 3.6).

Theorem C. Let $G$ be a finitely generated pro-p group containing an open subgroup $H$ which has a non-trivial decomposition as free product, i.e., there exists non-trivial closed subgroups $A, B \subsetneq H$ such that $H=A \amalg B$. Then $G$ is isomorphic to the pro-p fundamental group of a finite graph of pro-p groups with finite edge stabilizers.

Two achievements had caused dramatic advances in the combinatorial theory of groups; Bass-Serre theory of groups acting on trees and 'Stallings' decomposition theorem' of groups with infinitely many ends. The results of this paper contribute to the theory of pro- $p$ groups acting on pro- $p$ trees. Nevertheless, the absense of a 'Stallings' decomposition theorem' in the pro- $p$ context is still overshadowing the combinatorial theory of pro- $p$ groups.

\section{Preliminaries}

We will use the notion of graph as introduced by J-P. Serre in [9, §2.1].

2.1. Finite graphs of pro- $p$ groups. Let $\Gamma$ be a finite connected graph. A graph of groups $\mathcal{G}$ based on $\Gamma$ is called a finite graph of pro-p groups, if all vertex groups $\mathcal{G}(v), v \in V(\Gamma)$, and all edge groups $\mathcal{G}(e), e \in E(\Gamma)$, are pro- $p$ groups, and if all the group monomorphisms $\alpha_{e}: \mathcal{G}(e) \rightarrow \mathcal{G}(t(e))$ are continuous. So, if $(\mathcal{G}, \Gamma)$ is an (abstract) graph of groups such that all vertex and edge groups are finitely generated pro- $p$ groups, then by a theorem of J-P. Serre (cf. [5, §4.8]), $(\mathcal{G}, \Gamma)$ is a finite graph of pro- $p$ groups.

A finite graph of pro- $p$ groups $(\mathcal{G}, \Gamma)$ is said to be reduced, if for every geometric edge $\{e, \bar{e}\}$ which is not a loop neither $\alpha_{e}: \mathcal{G}(e) \rightarrow \mathcal{G}(t(e))$ nor $\alpha_{\bar{e}}: \mathcal{G}(e) \rightarrow \mathcal{G}(o(e))$ is an isomorphism. Any finite graph of pro- $p$ groups can be transformed in a reduced finite graph of pro- $p$ groups by the following procedure: If $\{e, \bar{e}\}$ is a geometric edge which is not a loop, we can remove $\{e, \bar{e}\}$ from the edge set of $\Gamma$, and identify $o(e)$ and $t(e)$ in a new vertex $y$. Let $\Gamma^{\prime}$ be the finite graph given by $V\left(\Gamma^{\prime}\right)=\{y\} \sqcup V(\Gamma) \backslash\{o(e), t(e)\}$ and $E\left(\Gamma^{\prime}\right)=E(\Gamma) \backslash\{e, \bar{e}\}$, and let $\mathcal{G}^{\prime}$ denote the finite graph of pro- $p$ groups based on $\Gamma^{\prime}$ given by $\mathcal{G}^{\prime}(y)=\mathcal{G}(o(e))$ if $\alpha_{e}$ is an isomorphism, and $\mathcal{G}^{\prime}(y)=\mathcal{G}(t(e))$ if $\alpha_{e}$ is not an isomorphism. This procedure can be continued until $\alpha_{e}$ is not surjective for all edges not defining loops. The resulting finite graph of pro- $p$ groups $\left(\mathcal{G}_{\text {red }}, \Gamma_{\text {red }}\right)$ is reduced.

2.2. The fundamental pro- $p$ group of a finite graph of finitely generated pro- $p$ groups. Let $(\mathcal{G}, \Gamma)$ be a finite graph of finitely generated pro- $p$ groups. We define the fundamental pro-p group $G=\Pi_{1}\left(\mathcal{G}, \Gamma, v_{0}\right), v_{0} \in V(\Gamma)$, of $(\mathcal{G}, \Gamma)$ to be the pro- $p$ completion of the usual fundamental group $\pi_{1}\left(\mathcal{G}, \Gamma, v_{0}\right)$ (cf. [9, §5.1]). In general, $\pi_{1}\left(\mathcal{G}, \Gamma, v_{0}\right)$ does not have to be residually $p$, but this will be the case in all of our considerations. In particular, edge and vertex groups will be subgroups of $\Pi_{1}\left(\mathcal{G}, \Gamma, v_{0}\right)$. Since $\mathcal{G}(e), \mathcal{G}(v)$ are finitely generated, by a theorem of J-P. Serre (cf. [5. §4.8]), our definition is equivalent to the original definition of the fundamental group of a graph of groups in the category of pro- $p$ groups (cf. [14]). Note that the previously mentioned reduction process does not change the fundamental pro- $p$ group, i.e., one has a canonical isomorphism $\Pi_{1}\left(\mathcal{G}, \Gamma, v_{0}\right) \simeq \Pi_{1}\left(\mathcal{G}_{\text {red }}, \Gamma_{\text {red }}, w_{0}\right)$. So, if the pro- $p$ group $G$ is the fundamental group of a finite graph of pro- $p$ groups, we may assume that the finite graph of pro- $p$ groups is reduced. 
2.3. The fundamental pro- $p$ group of a finite graph of finite $p$-groups. Let $(\mathcal{G}, \Gamma)$ be a finite graph of finite $p$-groups. By [14, Thm. 3.10], every finite subgroup of $G=\Pi_{1}\left(\mathcal{G}, \Gamma, v_{0}\right)$ is conjugate to a subgroup of a vertex group of $(\mathcal{G}, \Gamma)$. Hence $G$ has only finitely many finite subgroups up to conjugation. In particular, every maximal finite subgroup of $G$ is $G$-conjugate to a vertex group of $(\mathcal{G}, \Gamma)$, and the converse is true if $(\mathcal{G}, \Gamma)$ is a reduced finite graph of finite $p$-groups.

\section{ViRTUALLY FREE PRO- $p$ PRODUCTS}

3.1. Virtually free pro- $p$ groups. A pro- $p$ group $G$ will be called to be a free pro$p$ product if there exist non-trivial closed subgroups $A$ and $B$ such that $G=A \amalg B$. Otherwise we shall say that $G$ is $\amalg$-indecomposable. The following properties are well known.

Proposition 3.1. Let $H=\coprod_{i \in I} H_{i} \coprod F$ be a finitely generated pro-p group with $a \amalg$-decompositon, where $H_{i}$ are non-trivial $\amalg$-indecomposable pro-p-groups, and $F$ is a free pro-p group. Then

(a) $I$ is finite, and $H_{i}, i \in I$, and $F$ are finitely generated.

(b) Any finitely generated $\amalg$-indecomposable subgroup $A$ of $H$ is conjugate to a subgroup of $H_{i}$ for some $i \in I$. Moreover, if $H=A \amalg B$ for some closed subgroup $B$ of $H$, then $A$ is conjugate to some $H_{i}, i \in I$.

(c) $H_{i} \cap H_{j}^{h}=1$ if either $i \neq j$ or $h \notin H_{i}$.

(d) For $K \subseteq H_{i}, K \neq\{1\}$, one has $N_{H}(K) \subseteq H_{i}$. In particular, if $H_{i}$ is finite, so is $N_{H}(K)$.

Proof. (a) is obvious. The first statement of (b) follows from the pro- $p$ version of the Kurosh subgroup theorem [1, Thm. 4.4] and the second statement from [3, Thm. 4.3]. For (c) see Theorems 4.2 (a) and 4.3 (a) in 4. In order to prove (d) take $h \in N_{G}(K)$. Then $K \subseteq H_{i} \cap H_{i}^{h}$, and, by (c), one has $h \in H_{i}$.

From Proposition 3.1 one concludes the following properties for virtual free pro- $p$ products.

Proposition 3.2. Let $(\mathcal{G}, \Gamma)$ be a reduced finite graph of finite p-groups, and suppose that $G=\Pi_{1}\left(\mathcal{G}, \Gamma, v_{0}\right)$ contains an open, normal subgroup $H=F \amalg H_{1} \amalg \cdots \amalg H_{s}$, $H_{i} \neq\{1\}$, for some free pro-p subgroup $F$ of rank $r, 0 \leq r<\infty$, such that $r+s \geq 2$. Then one has the following.

(a) For any edge e of $\Gamma$ one has $\mathcal{G}(e) \cap H=\{1\}$; in particular, $|\mathcal{G}(e)| \leq|G: H|$.

(b) $|\mathbf{E}(\Gamma)| \leq 2(r+s)-1$ and $|V(\Gamma)| \leq 2(r+s)$, where $V(\Gamma)$ is the set of vertices of $\Gamma$, and $\mathbf{E}(\Gamma)$ is the set of geometric edges of $\Gamma$.

Proof. Let $X=\pi_{1}\left(\mathcal{G}, \Gamma, v_{0}\right)$ be the abstract fundamental group of the graph of groups and $Y=X \cap H$. Hence $G$ and $H$ are the pro- $p$ completions of $X$ and $Y$, respectively. Moreover, $|X: Y|=|G: H|$.

(a) Suppose that $\mathcal{G}(e) \cap H \neq\{1\}$. Since $H$ is normal in $G, N_{G}(\mathcal{G}(e))$ normalizes $\mathcal{G}(e) \cap H$. We claim that $N_{G}(\mathcal{G}(e))$ is infinite. One has to distinguish two cases: Case 1: $\{e, \bar{e}\}$ is not a loop. In this case $N_{G}(\mathcal{G}(e))$ contains the infinite group $\left\langle N_{\mathcal{G}(v)}(\mathcal{G}(e)), N_{\mathcal{G}(w)}(\mathcal{G}(e))\right\rangle$, where $v=o(e), w=t(e)$. Case 2: $\{e, \bar{e}\}$ is a loop. Let $v=t(e)=o(e)$, and let $z_{e} \in G$ be the stable letter associated with $e$. If $\mathcal{G}(e)=\mathcal{G}(v)$, then $N_{G}(\mathcal{G}(e))$ contains the infinite group $\left\langle z_{e}\right\rangle$. Otherwise $N_{G}(\mathcal{G}(e))$ contains the infinite group $\left\langle N_{\mathcal{G}(v)}(\mathcal{G}(e)), z_{e} N_{\mathcal{G}(v)}(\mathcal{G}(e)) z_{e}^{-1}\right\rangle$. 
Since $|G: H|<\infty$, the fact that $N_{G}(\mathcal{G}(e))$ is infinite implies that $N_{H}(\mathcal{G}(e) \cap H)=$ $N_{G}(\mathcal{G}(e) \cap H) \cap H$ is infinite as well contradicting Proposition 3.1(d). Hence one has $\mathcal{G}(e) \cap H=\{1\}$ as required.

(b) It suffices to show the first inequality. By [9, §2.6, Ex. 3], one has

$$
\begin{aligned}
-\chi_{X} & =\sum_{e \in \mathbf{E}(\Gamma)} \frac{1}{|\mathcal{G}(e)|}-\sum_{v \in V(\Gamma)} \frac{1}{|\mathcal{G}(v)|} \\
& =-\frac{1}{|X: Y|} \cdot \chi_{Y} \\
& =\frac{1}{|X: Y|} \cdot\left(r+s-1-\sum_{1 \leq i \leq s} \frac{1}{\left|H_{i}\right|}\right),
\end{aligned}
$$

where $\chi_{X}$ denotes the Euler characteristic of the finitely generated virtually free group $X$. Thus one obtains

$$
r+s-1 \geq|X: Y|\left(\sum_{e \in \mathbf{E}(\Gamma)} \frac{1}{|\mathcal{G}(e)|}-\sum_{v \in V(\Gamma)} \frac{1}{|\mathcal{G}(v)|}\right) .
$$

As $(\mathcal{G}, \Gamma)$ is reduced, for every edge $e$ in a maximal subtree $T$ of $\Gamma$ the edge group $\mathcal{G}(e)$ is isomorphic to a proper subgroup of $\mathcal{G}(t(e))$. Hence, $|\mathcal{G}(t(e))| \geq 2|\mathcal{G}(e)|$. Let $E^{+}(T)$ be an orientation of $T$ such that every vertex of $\Gamma$ except $v_{0} \in V(\Gamma)$ is the terminus of precisely one edge of $T$, and let $f \in E(T)$ be an edge satisfying $t(f)=v_{0}$. Taking into account that $|\mathbf{E}(T)|=|V(\Gamma)|-1$, one concludes from (a) that

$$
r+s-1 \geq \frac{1}{2} \cdot \sum_{e \in \mathbf{E}(\Gamma) \backslash\{f, \bar{f}\}} \frac{|X: Y|}{|\mathcal{G}(e)|} \geq \frac{1}{2} \cdot(|\mathbf{E}(\Gamma)|-1) .
$$

This yields the claim.

From Proposition 3.2 one concludes the following straightforward fact.

Corollary 3.3. Let $(\mathcal{G}, \Gamma)$ be a reduced finite graph of finite p-groups, and suppose that $G=\Pi_{1}\left(\mathcal{G}, \Gamma, v_{0}\right)$ contains a free open subgroup $H$ of rank $r \geq 2$. Then there exist finitely many reduced finite graphs of finite p-groups $\left(\mathcal{G}^{\prime}, \Gamma^{\prime}\right)$ up to isomorphism such that $G \simeq \Pi_{1}\left(\mathcal{G}^{\prime}, \Gamma^{\prime}, w_{0}\right)$.

Let $G=\Pi_{1}\left(\mathcal{G}, \Gamma, v_{0}\right)$ be the pro- $p$ fundamental group of a finite graph of finite $p$-groups, and let $U$ be an open and normal subgroup of $G$. Then, by construction, $\widetilde{U}=\operatorname{cl}\left(\left\langle U \cap \mathcal{G}(v)^{g} \mid g \in G, v \in V(\Gamma)\right\rangle\right)$ is a closed normal subgroup of $G$. By [6. Prop. 1.10], one has a natural decomposition of $G / \widetilde{U}$ as the pro- $p$ fundamental group $G / \widetilde{U}=\Pi_{1}\left(\mathcal{G}_{U}, \Gamma, v_{0}\right)$ of a finite graph of finite $p$-groups $\left(\mathcal{G}_{U}, \Gamma\right)$, where the vertex and edge groups satisfy $\mathcal{G}_{U}(x)=\mathcal{G}(x) \widetilde{U} / \widetilde{U}, x \in V(\Gamma) \sqcup E(\Gamma)$. Thus we have a morphism $\eta:(\mathcal{G}, \Gamma) \longrightarrow\left(\mathcal{G}_{U}, \Gamma\right)$ of graphs of groups such that the induced homomorphism on the pro- $p$ fundamental groups coincides with the canonical projection $\varphi_{U}: G \longrightarrow G / \widetilde{U}$.

Lemma 3.4. Let $G=\Pi_{1}\left(\mathcal{G}, \Gamma, v_{0}\right)$ be the pro-p fundamental group of a finite graph of finite p-groups, and let $H$ be an open normal subgroup of $G$ that decomposes as a free pro-p product $H=\coprod_{1 \leq i \leq s} H_{i} \amalg F$ of finite $p$-groups $H_{i}$ and a free pro-p group $F$. Let $U \subseteq H$ be an open normal subgroup of $G$ such that $U \cap H_{i} \neq H_{i}$ for every $i \in\{1, \ldots, s\}$. If $(\mathcal{G}, \Gamma)$ is reduced, then $\left(\mathcal{G}_{U}, \Gamma\right)$ is reduced. 
Proof. Suppose on the contrary that there exists an edge $e$ in $\Gamma$ which is not a loop such that for $v=t(e)$ one has $\mathcal{G}(v) \widetilde{U}=\mathcal{G}(e) \widetilde{U} \subseteq G / \widetilde{U}$. Then, by the second isomorphism theorem,

$$
\mathcal{G}(v)=\mathcal{G}(e)(\widetilde{U} \cap \mathcal{G}(v)) .
$$

As $(\mathcal{G}, \Gamma)$ is reduced, and thus $\mathcal{G}(e) \neq \mathcal{G}(v)$, one has $\widetilde{U} \cap \mathcal{G}(v) \neq\{1\}$. From Proposition 3.1 (a) one deduces that $\widetilde{U} \cap \mathcal{G}(v)$ is contained in some $H_{i}^{g}$ for $1 \leq i \leq s$ and $g \in G$. If $N_{G}(\widetilde{U} \cap \mathcal{G}(v))$ would be infinite, so would be $N_{H}(\widetilde{U} \cap \mathcal{G}(v))$ contradicting Proposition 3.1(d). Hence $N_{G}(\widetilde{U} \cap \mathcal{G}(v))$ is finite and equal to $\mathcal{G}(v)$. In particular, for $y \in \mathcal{G}(v)$ one concludes that $H_{i}^{g y} \cap H_{i}^{g} \neq\{1\}$. Hence, by Proposition 3.1(c), $H_{i}^{g y}=H_{i}^{g}$ and thus $\mathcal{G}(v) \subseteq N_{G}\left(H_{i}^{g}\right)$. The maximality of $\mathcal{G}(v)$ and the finiteness of $N_{G}\left(H_{i}^{g}\right)$ (cf. Prop. 3.1(d)) imply that $\mathcal{G}(v)=N_{G}\left(H_{i}^{g}\right)$. By construction, $\mathcal{G}(e) H_{i}^{g}$ is a finite subgroup of $G$ containing $\mathcal{G}(v)$ (cf. (3.4)). As $\mathcal{G}(v)$ is a maximal finite subgroup of $G$, this implies that

$$
\mathcal{G}(e)(\widetilde{U} \cap \mathcal{G}(v))=\mathcal{G}(v)=\mathcal{G}(e) H_{i}^{g} .
$$

Since $\widetilde{U} \cap \mathcal{G}(v) \subseteq H_{i}^{g}$, and as $\mathcal{G}(e) \cap H_{i}^{g}=\{1\}$ (cf. Prop. [3.2(a)), one concludes that $\widetilde{U} \cap \mathcal{G}(v)=H_{i}^{g}$. Hence $H_{i} \subseteq \widetilde{U} \subseteq U$ contradicting the hypothesis.

The proof of the structure theorem for virtual free pro- $p$ products (cf. Thm. (3.6) in the subsequent subsection is based on the following result due to W. Herfort and the second author.

Theorem 3.5. (cf. [2, Thm. 1.1]) Let $G$ be a finitely generated pro-p group with a free open subgroup $F$. Then $G$ is the pro-p fundamental group of a finite graph of finite p-groups whose orders are bounded by $|G: F|$.

3.2. Virtual free pro- $p$ products. The following theorem gives a description of the structure of virtual free pro- $p$ products.

Theorem 3.6. Let $G$ be a finitely generated pro-p group containing an open subgroup $H$ which has a non-trivial decomposition as free product, i.e., there exists non-trivial closed subgroups $A, B \subsetneq H$ such that $H=A \amalg B$. Then $G$ is isomorphic to the pro-p fundamental group of a finite graph of pro-p groups with finite edge stabilizers.

Proof. By replacing $H$ by the core of $H$ in $G$ and applying the Kurosh subgroup theorem for open subgroups (cf. [5, Thm. 9.1.9]), we may assume that $H$ is normal in $G$. Refining the free decomposition if necessary and collecting free factors isomorphic to $\mathbb{Z}_{p}$ we obtain a free decomposition

$$
H=F \amalg H_{1} \amalg \cdots \amalg H_{s},
$$

where $F$ is a free subgroup of rank $t$, and the $H_{i}$ are $\amalg$-indecomposable finitely generated subgroups which are not isomorphic to $\mathbb{Z}_{p}$ (cf. Prop. 3.1(a)). By hypothesis, $s+t \geq 2$. By construction, one has for all $g \in G$ and for all $i \in\{1, \ldots, s\}$ that $H_{i}^{g}$ is a free factor of $H$. Since $H_{i}$ is indecomposable, we deduce from Proposition 3.1 (b) that the indecomposable non-free subgroup $H_{i}^{g}$ of $H$ equals $H_{j}^{h}$ for some $j \in\{1, \ldots, s\}$. Thus $\left\{H_{i}^{g} \mid g \in G, 1 \leq i \leq s\right\}=\left\{H_{i}^{h} \mid h \in H, 1 \leq i \leq s\right\}$.

Step 1: Let $\mathcal{B}$ be a basis of neighbourhoods of $1_{G} \in G$ consisting of open normal subgroups $U$ of $G$ which are contained in $H$ with $H_{i} \not \subset U$ for every $i=1, \ldots s$. For 
$U \in \mathcal{B}$ put

$$
\widetilde{U}=\operatorname{cl}\left(\left\langle U \cap H_{i}^{g} \mid g \in G, 1 \leq i \leq s\right\rangle\right)=\operatorname{cl}\left(\left\langle U \cap H_{i}^{h} \mid h \in H, 1 \leq i \leq s\right\rangle\right) .
$$

Then $\widetilde{U}$ is a closed normal subgroup of $H$, and

$$
H / \widetilde{U}=F \amalg H_{1} \widetilde{U} / \widetilde{U} \amalg \cdots \amalg H_{s} \widetilde{U} / \widetilde{U}
$$

(cf. 3, Prop. 1.18]). The group $G / \widetilde{U}$ contains the open normal subgroup $H / \widetilde{U}$ which is a finitely generated, virtually free pro- $p$ group (since $U / \widetilde{U}$ is free pro- $p$ by Theorem 2.6 in [14]), and thus $G / \widetilde{U}$ is a finitely generated, virtually free pro- $p$ group.

Step 2: By Theorem 3.5, $G / \widetilde{U}$ is isomorphic to the pro- $p$ fundamental group $\Pi_{1}\left(\mathcal{G}_{U}, \Gamma_{U}, v_{U}\right)$ of a finite graph of finite $p$-groups. Using the procedure described in subsection 2.2 we may assume that $\left(\mathcal{G}_{U}, \Gamma_{U}\right)$ is reduced. Hence from now on we may assume that for every $U \in \mathcal{B}$ the vertex groups of $G / \widetilde{U}=\Pi_{1}\left(\mathcal{G}_{U}, \Gamma_{U}, v_{U}\right)$ are representatives of the $G / \widetilde{U}$-conjugacy classes of maximal finite subgroups. Note that by Proposition $3.2\left(\right.$ a), one has $\mathcal{G}_{U}(e) \cap H / \tilde{U}=1$.

Step 3: As explained before Lemma 3.4 for $V \subseteq U$ both open and normal in $G$ the decomposition $G / \widetilde{V}=\Pi_{1}\left(\mathcal{G}_{V}, \Gamma_{V}, v_{V}\right)$ gives rise to a natural decomposition of $G / \widetilde{U}$ as the fundamental group $G / \widetilde{U}=\Pi_{1}\left(\mathcal{G}_{V, U}, \Gamma_{V}, v_{V}\right)$ of a graph of groups $\left(\mathcal{G}_{V, U}, \Gamma_{V}\right)$. Moreover, by Lemma 3.4, if $\left(\mathcal{G}_{V}, \Gamma_{V}\right)$ is reduced, then $\left(\mathcal{G}_{V, U}, \Gamma_{V}\right)$ is reduced. Thus in this case one has a morphism $\eta:\left(\mathcal{G}_{V}, \Gamma_{V}\right) \longrightarrow\left(\mathcal{G}_{V, U}, \Gamma_{V}\right)$ of reduced graph of groups such that the induced homomorphism on the pro- $p$ fundamental groups coincides with the canonical projection $\varphi_{U V}: G / \widetilde{V} \longrightarrow G / \widetilde{U}$.

Step 4: By Proposition 3.2, the number $\left|V\left(\Gamma_{U}\right)\right|+\left|\mathbf{E}\left(\Gamma_{U}\right)\right|$ is bounded by $4(r+s)-1$. Therefore, by passing to a cofinal system $\mathcal{C}$ of $\mathcal{B}$ if necessary, we may assume that $\Gamma_{U}=\Gamma$ for each $U \in \mathcal{C}$. Then, by Corollary 3.3 , the number of isomorphism classes of finite reduced graphs of finite $p$-groups $\left(\mathcal{G}_{U}^{\prime}, \Gamma\right)$ which are based on $\Gamma$ and satisfy $G / \widetilde{U} \simeq \Pi_{1}\left(\mathcal{G}^{\prime}, \Gamma, v_{0}\right)$ is finite. Suppose that $\Omega_{U}$ is a set containing a copy of every such isomorphism class. For $V \in \mathcal{C}, V \subseteq U$, one has a map $\omega_{V, U}: \Omega_{V} \rightarrow \Omega_{U}$ (cf. Step 3). Hence $\Omega=\lim _{U \in \mathcal{C}} \Omega_{U}$ is non-empty. Let $\left(\mathcal{G}_{U}^{\prime}, \Gamma\right)_{U \in \mathcal{C}} \in \Omega$. Then $\left(\mathcal{G}^{\prime}, \Gamma\right)$ given by $\mathcal{G}^{\prime}(x)=\lim _{\mathcal{G}_{U}^{\prime}}^{\prime}(x)$ if $x$ is either a vertex or an edge of $\Gamma$, is a reduced finite graph of finitely generated pro- $p$ groups satisfying $G \simeq \Pi_{1}\left(\mathcal{G}^{\prime}, \Gamma, v_{0}\right)$. By Proposition $3.2(\mathrm{a}), \mathcal{G}^{\prime}(e)$ is finite for every edge $e$ of $\Gamma$. This yields the claim.

\section{REFERENCES}

[1] W. Herfort and L. Ribes, Subgroups of free pro-p-products, Math. Proc. Cambridge Philos. Soc. 101 (1987), no. 2, 197-206. MR 870590 (87m:20083)

[2] W. Herfort and P. A. Zalesskii, Virtually free pro-p groups, Publ. Math. Inst. Hautes Études Sci. 118 (2013), 193-211. MR 3150249

[3] O. V. Mel'nikov, Subgroups and the homology of free products of profinite groups, Izv. Akad. Nauk SSSR Ser. Mat. 53 (1989), no. 1, 97-120. MR 992980 (91b:20033)

[4] L. Ribes and P. A. Zalesskii, Pro- $p$ trees and applications, New horizons in pro- $p$ groups, Progr. Math., vol. 184, Birkhäuser Boston, Boston, MA, 2000, pp. 75-119. MR 1765118 (2001f:20057)

[5] _ Profinite groups, Ergebnisse der Mathematik und ihrer Grenzgebiete. 3. Folge. A Series of Modern Surveys in Mathematics [Results in Mathematics and Related Areas. 3rd Series. A Series of Modern Surveys in Mathematics], vol. 40, Springer-Verlag, Berlin, 2000. MR 1775104 (2001k:20060)

[6] L. Ribes and P. A. Zalesskiı̌, Normalizers in groups and their profinite completion, Rev. Mat. Iberoam. 30 (2014), no. 1, 167-192. 
[7] J-P. Serre, Sur la dimension cohomologique des groupes profinis, Topology 3 (1965), 413-420. MR 0180619 (31 \#4853)

[8] _ Cohomologie des groupes discrets, Prospects in mathematics (Proc. Sympos., Princeton Univ., Princeton, N.J., 1970), Princeton Univ. Press, Princeton, N.J., 1971, pp. 77-169. Ann. of Math. Studies, No. 70. MR 0385006 (52 \#5876)

[9] — Trees, Springer-Verlag, Berlin, 1980, Translated from the French by John Stillwell. MR 607504 (82c:20083)

[10] J. R. Stallings, Groups of dimension 1 are locally free, Bull. Amer. Math. Soc. 74 (1968), 361-364. MR 0223439 (36 \#6487)

[11] _ On torsion-free groups with infinitely many ends, Ann. of Math. (2) 88 (1968), $312-334$.

[12] _ Group theory and three-dimensional manifolds, Yale University Press, New Haven, Conn.-London, 1971, A James K. Whittemore Lecture in Mathematics given at Yale University, 1969, Yale Mathematical Monographs, 4. MR 0415622 (54 \#3705)

[13] R. G. Swan, Groups of cohomological dimension one, J. Algebra 12 (1969), 585-610. MR 0240177 (39 \#1531)

[14] P. A. Zalesskiı̆ and O. V. Mel'nikov, Subgroups of profinite groups acting on trees, Mat. Sb. (N.S.) 135(177) (1988), no. 4, 419-439, 559. MR 942131 (90f:20041)

Th. Weigel, Università di Milano-Bicocca, U5-3067, Via R.Cozzi, 55, 20125 Milano, ITALY

E-mail address: thomas.weigel@unimib.it

P. A. ZalesskiĬ, Department of Mathematics, University of Brasilia, 70.910 Brasilia DF, BRAZIL

E-mail address: pz@mat.unb.br 